\title{
Alternativas orgánicas para el logro de producciones más limpias de la fresa en Pamplona, Norte de Santander
}

\section{Organic alternatives for the achievement of cleaner strawberry production in Pamplona, North of Santander}

\author{
DOI: http://doi.org/10.17981/ingecuc.1612.2020.14 \\ Artículo de Investigación Científica. Fecha de Recepción: 30/01/2020. Fecha de Aceptación: 02/03/2020 \\ Leónides Castellanos González (i) \\ Universidad de Pamplona. Norte de Santander (Colombia) \\ lclcastell.@gmail.com \\ Nestor Céspedes Novoa \\ Granja Agrobiológica Sol Vida, ASPAGRO, Pamplona. Norte de Santander (Colombia) \\ nestorcespedeshse@gmail.com. \\ Alex Baldovino Sanjuán \\ Universidad de Pamplona. Norte de Santander (Colombia) \\ alexbaldovino27@hotmail.com
}

Para citar este artículo:

L. Castellanos Gonález, N. Céspedes Novoa y A. Baldovino Sanjuán, "Alternativas orgánicas para el logro de producciones más limpias de la fresa en Pamplona, Norte de Santander”, INGE CUC, vol. 16, no. 1, pp. 187-196, 2020. DOI: http://doi.org/10.17981/ingecuc.1612.2020.14

\section{Resumen}

Introducción- El cultivo de fresa es el tercero en importancia en Pamplona (Norte de Santander, Colombia). Las plantaciones reciben aplicaciones indiscriminadas de fertilizantes y plaguicidas químicos.

Objetivo- Evaluar alternativas orgánicas para el control de las enfermedades foliares y del suelo, las babosas, y para la carencia de fósforo con vistas al logro de producciones más limpias de la fresa.

Metodología- Se desarrollaron cuatro ensayos en campos de fresa; uno para el control de enfermedades de la parte aérea, el segundo para el control de enfermedades de la raíz, el tercero para la nutrición fosfórica y el cuarto para el control de babosas. En los tres primeros se evaluaron diferentes biopreparados producidos localmente a partir de microorganismos eficientes y en el último la tierra de diatomeas.

Resultados- Caldo Rizósfera y Microorganismos Eficientes (ME) mostraron resultados similares al fungicida protectante Dithane contra mancha por Ramularia, la antracnosis en hojas, flores y frutos, la pudrición por Botrytis y la mancha bacteriana, mientras que Caldo Rizósfera, ME y otra variante de ME (M6) resultaron similares al fungicida Benomil y fosfito de potasio para el control de las enfermedades radiculares causadas por Fusaium spp y Phytophthora fragarie. Caldo Rizósfera y M6 disminuyeron en mayor medida la incidencia y severidad de la deficiencia de fósforo y favorecieron una mayor concentración de este en las hojas. La tierra de diatomea demostró eficacia para el control de las babosas con dos aplicaciones, dosis entre 4 y $8 \mathrm{~kg} / \mathrm{ha}$ y la adición de PEGAL $\mathrm{CS} \mathrm{pH} \mathrm{18,767} \mathrm{g/L} \mathrm{como} \mathrm{adherente.}$

Conclusiones- Los resultados permiten incorporar nuevas alternativas para el control de enfermedades foliares y radiculares, así como las babosas y la nutrición fosfórica en el cultivo de la fresa en aras de obtener producciones más limpias en las condiciones de Pamplona (Norte de Santander, Colombia).

Palabras clave - Fragaria $\times$ ananassa; microorganismos antagonistas; plaguicidas alternativos; biofertilizantes; nutrición fosfórica; medio ambiente

\section{Abstract}

Introduction- The strawberry crop is the third in importance in Pamplona (North of Santander, Colombia). The plantations receive indiscriminate applications of fertilizers and chemical pesticides.

Objective- To evaluate organic control alternatives for foliar and soil diseases, slugs, and for the lack of phosphorus with a view to achieving cleaner strawberry production

Methodology- Four trials were carried out in strawberry fields; one for the control of diseases of the aerial part, the second for the control of root diseases, the third for phosphoric nutrition and the fourth for the control of slugs. In the first three different biopreparations produced locally from efficient microorganisms were evaluated and, in the latter, the diatomaceous earth.

Results- The Rhizosphere Broth (RB) and MM products showed similar results to the Dithane protective fungicide against Ramularia spot, the anthracnose on leaves, flowers and fruits, Botrytis rot and bacterial spot, while, MM and another variant of MM (M6) were similar to the fungicide Benomil and potassium phosphite for the control of root diseases caused by Fusarium spp. and Phytophthora fragarie. RB and M6 decreased the incidence and severity of phosphorus deficiency and favored a higher concentration of phosphorus in the leaves. Diatomaceous earth demonstrated efficacy for the control of slugs with two applications, at doses between 4 and $8 \mathrm{~kg} / \mathrm{ha}$.

Conclusions- The results allow incorporating new alternatives for the control of foliar and root diseases, as well as slugs and phosphoric nutrition in the cultivation of strawberries in order to obtain cleaner productions under the conditions of Pamplona (Norte de Santander, Colombia).

Keywords - Fragaria $\times$ ananassa; antagonist microorganism; alternative pesticides; biofertilizers; phosphoric nutrition; environment 


\section{INTRODUCCIÓN}

El departamento Norte de Santander (Colombia) ocupa el tercer lugar en la producción de fresa Fragaria $\times$ ananassa (Duchesne ex Weston) Duchesne ex Rozieren Colombia [1], siendo el cultivo el tercer renglón agrícola del municipio de Pamplona.

El cultivo de fresa se ve afectado en Colombia por numerosas plagas y enfermedades. Las siguientes son las enfermedades más comunes del cultivo de fresa a nivel mundial: pudrición de la corona (Phytophthora fragariae Hichman), marchitez por Verticillium (Verticillium dahliae Kleb), pudrición de las raíces por Rhizoctonia sp. y marchitez por Fusarium (Fusarium oxysporum Schlencht.), podredumbre gris (Botrytis cinerea (De Bary) Whetze) y mancha foliar común (Ramularia tulasnei Fuckel), antracnosis (Colletotrichum gloeosporoides Penz.) y oidio (Oidium sp.). Como podredumbres poscosecha, se informan las siguientes: podredumbre ácida, Geotrichum sp., podredumbre blanda de Rhizopus sp., moho verde, Penicillium sp. y moho gris, Botrytis cinerea [2]. La fresa tiene altas características nutricionales, medicinales y de valor económico sin embargo es altamente susceptible al ataque de patógenos, por lo cual, uno de los principales retos en el desarrollo del cultivo e incluso en la poscosecha de la fruta, es el manejo de las enfermedades [3].

Las plagas de insectos como Aphis spp., Trips (Frankiniella spp.), moscas blancas (Trialeurodes vaporariorum Westwood), moscas de la fruta (Anastrepha spp.) y el gusano cortador (Spodoptera sp.) son plagas importantes del follaje de la fresa, así como las plagas de ácaros, entre ellas, el ácaro blanco Steneotarsonemus pallidus Banks y los ácaros rojos (Tetranychus spp.). Las plagas de insectos más importantes de la raíz son Anomala spp., Phyllophaga spp. y Cyclocephala spp. [1].

Algunas babosas también son plagas importantes por su daño a las fresas como Milax gagates Draparnaut. Se ha informado que otras especies de babosas causaron daños en las frutas de fresa en Pamplona [4].

El fósforo $(\mathrm{P})$ es uno de los principales macronutrientes esenciales requeridos por todos los organismos vivos, pero también es uno de los elementos menos disponibles de la rizosfera, participando en procesos estructurales, enzimáticos y energéticos de las plantas [5]. La aplicación de una fuente de materia orgánica rica en microorganismos al suelo influyó en la actividad enzimática y el contenido de $\mathrm{P}$ disponible en el suelo, reflejado en una acumulación diferencial de $\mathrm{P}$ en las plantas [6].
La deficiencia de fósforo en la planta de fresa disminuye la producción, las hojas más viejas toman colores marrones rojizo, se vuelven necróticas y caen prematuramente. Las frutas son pequeñas y su maduración se retrasa, alterando sus propiedades organolépticas, volviéndolas más ácidas. Cuando el suelo carece de fósforo disponible, la cosecha de fresa puede reducirse en un 50\% y el contenido de vitaminas disminuye [7]. La falta de fósforo es otro de los problemas de la fresa en Pamplona debido al predominio de suelos con $\mathrm{pH}$ bajo.

Debido a esto, uno de los grandes problemas en el cultivo de fresa, en Colombia, es la cantidad de pesticidas y fertilizantes químicos que se aplican con un alto riesgo para los consumidores cuando las frutas de fresa se comercializan frescas. Por otro lado, se desconocen los niveles de residuos de pesticidas y, dado que no hay agua de calidad para los cultivos, se obtienen fresas contaminadas que causan enfermedades transmitidas por los alimentos [8].

Se han realizado muchos esfuerzos en Colombia con el objetivo de reducir el uso indiscriminado de agroquímicos en las actividades agrícolas. Sin embargo, en el año 2007, según el Instituto Nacional de Salud Pública se informaron las intoxicaciones por pesticidas más altas en Norte de Santander en los últimos diez años [9].

Existen muchas alternativas biológicas que pudieran aplicarse para disminuir el número de tratamientos de fungicidas químicos. Entre las posibles alternativas menciona a los antagonistas Trichoderma spp., Clonostachys rosea, Streptomyces platensis, la aplicación de hongos micorrícicos arbusculares o de bacterias y levaduras promotoras del crecimiento vegetal que hacen efecto antagonista o confieren a la planta resistencia inducida [3].

Los Microorganismos Eficientes (ME) son una combinación de microorganismos beneficiosos de cuatro grupos principales: bacterias fototróficas, levaduras, bacterias productoras de ácido láctico y hongos de fermentación obtenidos de ecosistemas vírgenes, que tienen efecto contra bacterias y hongos fitopatógenos, actúan como fertilizante foliar y como mejoradores de la fertilidad del suelo por fijar nitrógeno atmosférico y solubilizar el fósforo [10].

Los Microorganismos de Montaña (MM) se podrían catalogar como un tipo de ME, o sea, un consorcio de microbianos que se obtiene de la rizósfera de las plantas y producen los mismos beneficios que los ME [11], mientras que el caldo rizósfera es otro bioproducto obtenido por vía fermentativa a partir de las raíces de algunas plantas con adición 
de yogurt y otros productos naturales como la roca fosfórica, que incrementa microrganismos beneficiosos y se recomienda con los mismos fines que los microrganismos eficiente (MM) [12].

La Asociación de Productores Agropecuarios de Pamplona (ASPAGRO) produce y comercializa algunos bioproductos artesanales a base de microrganismos eficientes que podrían ayudar a establecer una tecnología para la producción más limpia de la fresa en esta región, pero no se han realizado investigaciones científicas que verifiquen su eficiencia para el control de enfermedades, ni para solubilizar el fósforo, ni tampoco el empleo de la tierra de diatomea para el control de las babosas, otro problema serio del cultivo.

Por tal razón se propuso un proyecto que tuvo como objetivo evaluar alternativas orgánicas con biopreparados y la tierra de diatomea contra los principales agentes nocivos del cultivo para el logro de producciones más limpias de la fresa en Pamplona, Norte de Santander.

\section{Antecedentes}

Las tecnologías limpias consisten en la aplicación de estrategias que incluyen técnicas tales como reciclado, sustitución, recuperación y revalorización. Pueden ser muy sencillas, pues se puede tratar de un simple cambio en un procedimiento, o pueden ser sofisticadas. Entre las ventajas de la aplicación de tecnologías limpias se encuentran: mejores resultados al no crear contaminación, sistemas implantados son permanentes, fiables, eficaces, de gran rendimiento, rentables y casi siempre aumentan la productividad e implican ahorros considerables, así como la prevención implica una ventaja económica evidente en comparación con la descontaminación [13].

Un estudio realizado en España sobre la huella ecológica del cultivo de la fresa puso de manifiesto que los fertilizantes seguidos por los pesticidas son los que más aportan a la contaminación ambiental, por lo que recomiendan llevar a cabo una reducción y ajuste de los fertilizantes y manejo de pesticidas [14].

En otro experimento desarrollado en Perú se verificó que los mejores rendimientos en fresa se lograron para la variedad Selva en el tratamiento de la interacción de bocashi (compuesto orgánico fermentado) y microorganismos eficientes al $1 \%$ [15].

En el departamento de Boyacá se ha investigado sobre la fertilización orgánica de la fresa comprándose diferentes proporciones de cascarilla de arroz y fibra de coco verificándose como mejor alternativa la fibra de coco a la proporción del 50\% [16].

Otro estudio comparara dos tipos de fertilización (orgánica y química) en la parroquia Octavio Cordero Palacios (Cantón Cuenca). Los tratamientos orgánicos empleados fueron fertivegetal, biol de hiervas, té de estiércol bovino, humus líquido y purín de cerdo, aplicados en las diferentes etapas del cultivo comparados con la fertilización química estándar. Los resultados fueron alentadores en las variables de rendimientos y sus componentes, aunque se consideró que estos podían ser mejorados [17].

En Ecuador se probaron tres fungicidas biológicos en el cultivo de la fresa a base de Trichoderma, otro de Bacillus subtilis y un complejo biológico a base de bacterias benéficas y se verificó similar nivel de severidad de Botrytis cinerea con los productos biológicos que con el fungicida químico. En la investigación se evaluaron cepas de Trichoderma spp. contra $B$. cinérea en fresa. Los resultados mostraron una reducción de la incidencia de la enfermedad con $T$. harzianum y $T$. lignorum y al mismo tiempo los tratamientos con Trichoderma redujeron significativamente la severidad de la enfermedad [18].

En otra investigación desarrollada en Cundinamarca se compararon la aplicación de dos fungicidas de síntesis química (propiconazole y difenoconazol), en relación con dos productos biológicos a base de Trichoderma lignorum y Saccharomyces cerevisiae para el control de la pudrición gris de los frutos $B$. cinérea no encontrándose diferencias significativas en cuanto a la producción de fruto de fresa sano ente los productos químicos y los biológicos [19].

\section{Metodología}

Se desarrollaron cuatro ensayos en campos de fresa, uno para el control de enfermedades de la parte aérea, el segundo para el control de enfermedades de la raíz, el tercero para favorecer la nutrición fosfórica y el cuarto para el control de babosas.

En los tres primeros se evaluaron diferentes biopreparados producidos localmente a partir de microorganismos eficientes (Tabla 1).

Los tratamientos contaron con algunos de estos biopreparados, solo o combinados entre si o con manejo de saneamiento para el caso de las enfermedades aéreas de la fresa, siempre comparados con uno o más plaguicidas o fertilizantes químicos usados en la producción y con un testigo o control sin tratamiento alguno, como aparece en cada tabla de resultados. 
TAbla 1. Descripción de cada BIopreparados de ASPAGRO utilizados, Contenido de Bacterias Solubilizadoras DE FÓSFORO (.B.SF) Y CONCENTRACIÓN DE UNIDADES FORMADORAS DE COLONIAS (UCF).

\begin{tabular}{|c|c|c|c|}
\hline $\begin{array}{l}\text { Biopreparados } \\
\text { comerciales }\end{array}$ & Ingredientes & $\begin{array}{l}\text { Tipo de } \\
\text { fermentación }\end{array}$ & $\begin{array}{c}\text { Uso Propuesto y } \\
\text { Concentración (UFC) }\end{array}$ \\
\hline $\mathrm{P} 1$ & Vinagre, roca fosfórica y melaza. & aeróbica & $\begin{array}{l}\text { Biofertilizante con B.S.F. } \\
8,34 \times 10^{5} \mathrm{UCF} / \mathrm{mL}\end{array}$ \\
\hline $\mathrm{P} 2$ & M, Eficientes de una zona virgen y melaza, ceniza y roca fosfórica. & anaeróbica & $\begin{array}{l}\text { Biofertilizante con B.S.F. } \\
\text { y antagonista } \\
3.2 \times 10^{5} \mathrm{UCF} / \mathrm{mL}\end{array}$ \\
\hline MM & Microorganismos de montaña, melaza y salvado de arroz (Oriza sativa L.). & aeróbica & $\begin{array}{l}\text { Biofertilizante con B.S.F. } \\
\text { y antagonista } \\
1,72 \times 10^{6} \mathrm{UFC} / \mathrm{mL}\end{array}$ \\
\hline Caldo rizósfera & $\begin{array}{l}\text { Raíces de plantas: ortiga (Urtica dioica L.), borraja (Borago officinalis } \\
\text { L.), kikuyo (Pennisetum clandestinum Hochts ex Chiov), trébol blanco } \\
\text { (Trifolium repens L.) conseguidas en la granja, yogurt, melaza, agua } \\
\text { oxigenada y harina de frijol (Phaseolus vulgaris L.). }\end{array}$ & aeróbica & $\begin{array}{l}\text { Biofertilizante con B.S.F. } \\
\text { y antagonista } \\
1,24 \times 10^{6} \mathrm{UFC} / \mathrm{mL}\end{array}$ \\
\hline $\begin{array}{l}\text { Biopreparado } \\
\text { de meconio de } \\
\text { terenero }\end{array}$ & $\begin{array}{l}\text { Meconio de ternero, agua, melaza y ahuyama (Cucurbita máxima Duch.) } \\
\text { cocida. }\end{array}$ & anaeróbica & $\begin{array}{l}\text { Biofertilizante con B.S.F. } \\
\text { y antagonista } \\
1,3 \times 10^{6} \mathrm{UFC} / \mathrm{mL}\end{array}$ \\
\hline $\begin{array}{l}\text { M. } \\
\text { Eficientes (M6) }\end{array}$ & $\begin{array}{l}\text { M. Eficientes de una zona virgen, vinagre, etanol, plantas aromáticas, } \\
\text { jengibre (Zingiber officinale Rosc.), ajo (Allium sativum L.), cebolla } \\
\text { (Allium cepa L.), pimienta y ají (Capsicum annuum L.). }\end{array}$ & anaeróbica & $\begin{array}{l}\text { Biofertilizante con B.S.F. } \\
\text { y antagonista } \\
9,13 \times 10^{5} \mathrm{UFC} / \mathrm{mL}\end{array}$ \\
\hline
\end{tabular}

En cada parcela o unidad experimental se determinó el porcentaje de incidencia de las enfermedades foliares radiculares de la fresa según el caso (plantas y órganos) y la severidad para las enfermedades del follaje de con las siguientes fórmulas [20]:

$$
\begin{aligned}
& \% \text { de incidenciaodistribucion }=\frac{n(\text { plantasafectadas })}{N(\text { evaluadas })} X 100 \\
& \% S(\text { severidad })=\frac{\Sigma(a X b)}{K N} X 100
\end{aligned}
$$

Al final del experimento se determinó el Área Bajo la Curva de Progreso de la Enfermedad (ABCPE) de cada parcela según el método propuesto por [21], para lo cual se utilizó (3):

$$
\mathbf{A B C P E}=\boldsymbol{\Sigma}\left[\left(\mathrm{X}_{i}+\mathrm{X}_{i}+1\right) / 2\right] *\left(\mathrm{~T}_{\mathrm{i}}+1-\mathrm{Ti}\right)
$$

En el experimento para determinar la acción solubilizadora de $\mathrm{P}$ por los biopreparados locales se determinaron las variables incidencia de los síntomas y severidad de carecía de P utilizando (1) y (2).

A los 150 días de montado el experimento se realizó un análisis del contenido de $\mathrm{P}$ foliar tomando una muestra representativa de cada parcela la cual fue llevada al Laboratorio de Química de la Universidad de Pamplona. Para determinar el contenido de fósforo del área foliar se procedió a recolectar 60 hojas en 60 plantas (la tercera hoja bajando) en de cada réplica de los ocho tratamientos, luego se llevó al horno por una semana, y se procedió hacer un molido o macerado de cada muestra foliar. Posteriormente se pesaron 2,5 g de muestra macerada y se llevaron a un crisol y se rea- lizó un previo quemado con la ayuda de un mechero, luego se llevaron las muestras a la mufla a $350^{\circ} \mathrm{C}$.

Cuando las muestras estaban de nuevo a temperatura ambiente se agregaron $25 \mathrm{ml}$ de ácido clorhídrico a cada crisol, se procedió a evaporar en una plancha de calentamiento, hasta que quedaran $10 \mathrm{ml}$ de la solución, luego se filtró cada muestra con la ayuda de papel filtro en tubos de ensayo, agregándole a cada muestra $3 \mathrm{ml}$ de solución A para identificación de concentración de área foliar de fósforo y $5 \mathrm{ml}$ de solución $\mathrm{B}$. Se agitó cada muestra hasta que tomó un color azul.

Finalmente, el resultado de cada muestra se llevó al fotocolorímetro de marca HACH (DR 3800) para el caso de fósforo de espectrofotómetro 489 fosfato, para obtener la lectura del contenido de $\mathrm{P}$ en $\mathrm{mg} / \mathrm{L}$, para luego hacer los cálculos para estimar las partes por millón (ppm) que tenía cada muestra por medio de (4).

$$
\operatorname{ppm}(m g / k g)=\frac{\text { pesodelamuestra }}{m g / \text { Ldecadamuestra }} * 1000
$$

En el último experimento se evaluó la eficacia de la Tierra de Diatomeas (TD) para el control de babosas en condiciones de campo. Se emplearon cinco tratamientos, tres de TD: $2 \mathrm{~kg} / \mathrm{ha}, 4 \mathrm{~kg} / \mathrm{ha}$ y $8 \mathrm{~kg} / \mathrm{ha}$, uno con cal agrícola $4 \mathrm{~kg} / \mathrm{ha}$ (estándar) y un testigo sin tratamiento. Se realizaron dos asperjaciones de los productos con diferencia de 7 días una de otra y en la segunda se adicionó el adherente de uso agrícola PEGAL CS a razón de alcohol graso etoxilado18, $767 \mathrm{~g} / \mathrm{L}$. Se realizó un monitoreo con trampas de $25 \times 25 \mathrm{~cm}$ antes y después de las aplicaciones de TD para determinar el número de babosas por trampas por trampa y estimar el porcentaje de mortalidad en cada tratamiento. 
Para todos los experimentos se utilizó un diseño de bloque al azar con diferentes tratamientos establecidos y cuatro réplicas. Las unidades experimentales (réplicas) fueron parcelas de plantaciones de fresa de entre un año y año y medio de la variedad Albión de al menos $20 \mathrm{~m}^{2}$, en la vereda Monteadentro del Municipio Pamplona, a una altura entre 2400 y 2600 m.s.n.m.

Los datos de las variables evaluadas se sometieron a un análisis de varianza para verificar la Hipótesis nula de que las medias eran iguales con previa verificación del supuesto de normalidad del modelo por la prueba de Kolmogorov Smirnov. Los datos de las variables en porcentajes se transformaron en 2 arcsen $\sqrt{ } \% / 100$. Las medias se compararon por la prueba de Tukey considerando un nivel de confianza del 95\%. Se utilizó el paquete estadístico SPSS para Windows versión 21.

\section{Resultados}

No hubo diferencia significativa entre los tratamientos para la variable incidencia de la enfermedad fungosa $R$. tulasnei, mientras que la severidad de la enfermedad se redujo por el tratamiento MM (que alcanzó 22\%) con respecto al testigo sin sanear, pero no con respecto al resto de los tratamientos. El área bajo la curva de progreso de la enfermedad (ABCPE) de la incidencia fue menor para los biopreparados MM, Caldo Rizósfera y el fungicida químico Dithane, mientras que para la severidad el ABCPE, fue menor para MM (Tabla 2).

TABLA 2. REsultado DEL ANÁLisis ESTADÍSTICO PARA LA ENFERMEDAD CAUSADA POR EL HONGO RAMULARIA Tulasnei Fuckel (Mycosphaerella fragariae (Tul.)) EN HOJAS DE FRESA SEGÚN LOS DIFERENTES TRATAMIENTOS

\begin{tabular}{|l|l|l|l|l|}
\hline \multicolumn{1}{|c|}{ Tratamientos } & $\begin{array}{c}\text { Incidencia } \\
(\%)\end{array}$ & $\begin{array}{c}\text { Severidad } \\
(\%)\end{array}$ & $\begin{array}{c}\text { ABCPE } \\
\text { (INC). }\end{array}$ & $\begin{array}{c}\text { ABCPE } \\
(\text { SEV). }\end{array}$ \\
\hline T. saneado & $50 \mathrm{a}$ & $27 \mathrm{ab}$ & $67,81 \mathrm{bc}$ & $23,59 \mathrm{c}$ \\
\hline Biopreparado P1 & $55 \mathrm{a}$ & $28 \mathrm{ab}$ & $71,31 \mathrm{ab}$ & $25,69 \mathrm{~b}$ \\
\hline Biopreparado P2 & $52 \mathrm{a}$ & $27 \mathrm{a}$ & $69,82 \mathrm{~b}$ & $26,56 \mathrm{~b}$ \\
\hline $\begin{array}{l}\text { M. de Montaña } \\
\text { (MM) }\end{array}$ & $51 \mathrm{a}$ & $22 \mathrm{~b}$ & $59,85 \mathrm{~d}$ & $19,84 \mathrm{~d}$ \\
\hline C. Rizósfera (CR) & $48 \mathrm{a}$ & $23 \mathrm{ab}$ & $63,61 \mathrm{~cd}$ & $21,84 \mathrm{c}$ \\
\hline $\begin{array}{l}\text { Biopreparado } \\
\text { meconio }\end{array}$ & $50 \mathrm{a}$ & $26 \mathrm{ab}$ & $70,35 \mathrm{~b}$ & $25,67 \mathrm{~b}$ \\
\hline M. Eficientes (M6) & $50 \mathrm{a}$ & $27 \mathrm{ab}$ & $68,33 \mathrm{bc}$ & $26,51 \mathrm{~b}$ \\
\hline Fungicida Dithane & $51 \mathrm{a}$ & $25 \mathrm{ab}$ & $67,02 \mathrm{bc}$ & $23,59 \mathrm{c}$ \\
\hline Testigo sin sanear & $51 \mathrm{a}$ & $28 \mathrm{a}$ & $77,17 \mathrm{~b}$ & $31,04 \mathrm{a}$ \\
\hline Coefici. Variac. (\%) & 23 & 10 & 10 & 20 \\
\hline Error típico* & 0,041 & 0.01 & 1,3 & 3,21 \\
\hline
\end{tabular}

* Letras desiguales en las columnas indican diferencia estadísticamente significativa por la prueba de Tukey.

Aunque desde el punto de vista estadístico se observaron diferencia estadística entre los tratamientos la enfermedad alcanzó un $22 \%$ de severidad en las hojas lo cual mantiene un alto potencial de inóculo en estos órganos con alto riesgo para la infección en los frutos y las flores, sobre todo si no se acompaña el proceso del cultivo con labores de saneamiento, actividad que encarece el cultivo y no se realiza por los agricultores.

No hubo diferencia estadísticamente significativa entre los tratamientos para la variable incidencia de la enfermedad fungosa antracnosis en las hojas de fresa, mientras que la severidad de la enfermedad se redujo con respecto al testigo con varios tratamientos con los mejores resultados para los tratamientos MM (que alcanzó $22 \%$ ) y Caldo Rizósfera (24\%). El ABCPE de la incidencia fue menor para los biopreparados MM, Caldo Rizósfera aunque otros tratamientos como P2 y el fungicida químico Dithane no se diferenciaron de estos, mientras que para la severidad el ABCPE, fue menor para MM y Caldo Rizósfera (Tabla 3).

TABla 3. Resultado DEL ANÁLisis ESTADÍSTICO PARA LA ENFERMEDAD ANTRACNOSIS CAUSADA POR EL HONGO COLlETOTRICHUM GLOEOSPORIOIDES EN HOJAS DE FRESA SUGÚN LOS DIFERENTES TRATAMIENTOS.

\begin{tabular}{|l|l|l|l|l|}
\hline \multicolumn{1}{|c|}{ Tratamientos } & \multicolumn{1}{|c|}{$\begin{array}{c}\text { Incidencia } \\
(\%)\end{array}$} & $\begin{array}{c}\text { Severidad } \\
(\%)\end{array}$ & $\begin{array}{c}\text { ABCPE } \\
(\text { INC). }\end{array}$ & $\begin{array}{c}\text { ABCPE } \\
(\text { SEV). }\end{array}$ \\
\hline T. saneado & $51 \mathrm{a}$ & $25 \mathrm{~cd}$ & $62,65 \mathrm{ab}$ & $21,73 \mathrm{~cd}$ \\
\hline Biopreparado P1 & $50 \mathrm{a}$ & $28 \mathrm{ab}$ & $59,15 \mathrm{bcd}$ & $23,01 \mathrm{bc}$ \\
\hline Biopreparado P2 & $50 \mathrm{a}$ & $27 \mathrm{abc}$ & $57,22 \mathrm{cde}$ & $22,57 \mathrm{bcd}$ \\
\hline $\begin{array}{l}\text { M. de Montaña } \\
\text { (MM) }\end{array}$ & $48 \mathrm{a}$ & $22 \mathrm{e}$ & $54,16 \mathrm{e}$ & $18,51 \mathrm{f}$ \\
\hline $\begin{array}{l}\text { C. Rizósfera } \\
\text { (CR) }\end{array}$ & $52 \mathrm{a}$ & $24 \mathrm{de}$ & $55,03 \mathrm{de}$ & $19,72 \mathrm{ef}$ \\
\hline $\begin{array}{l}\text { Biopreparado } \\
\text { meconio }\end{array}$ & $50 \mathrm{a}$ & $27 \mathrm{abc}$ & $59,15 \mathrm{bcd}$ & $22,73 \mathrm{bcd}$ \\
\hline $\begin{array}{l}\text { M. Eficientes } \\
\text { (M6) }\end{array}$ & $47 \mathrm{a}$ & $27 \mathrm{abcd}$ & $60,20 \mathrm{abc}$ & 23,8700 \\
\hline $\begin{array}{l}\text { Fungicida } \\
\text { Dithane }\end{array}$ & $50 \mathrm{a}$ & $25 \mathrm{bcd}$ & $57,75 \mathrm{cde}$ & $21,10 \mathrm{de}$ \\
\hline $\begin{array}{l}\text { Testigo sin } \\
\text { sanear }\end{array}$ & $50 \mathrm{a}$ & $29 \mathrm{a}$ & $64,22 \mathrm{a}$ & $28,29 \mathrm{a}$ \\
\hline $\begin{array}{l}\text { Coefici. Variac. } \\
\text { (\%) }\end{array}$ & 17 & 9 & 6 & 9 \\
\hline Error Típico* & 0,04 & 0,01 & 0,9 & 0,35 \\
\hline
\end{tabular}

* Letras desiguales en las columnas indican diferencia estadísticamente significativa por la prueba de Tukey.

Aunque desde el punto de vista estadístico en el control de la antracnosis en las hojas se observaron diferencia estadística de los productos alternativos y el fungicida químico con relación al testigo, la enfermedad alcanzó entre 22 y $24 \%$ de severidad en las hojas con los mejores tratamientos.

La incidencia de la antracnosis en los frutos de fresa se redujo con respecto al testigo sin sanear en varios tratamientos siendo los más destacados el testigo saneado, el biopreparado M6 y el fungicida Dithane, pero la incidencia en frutos en estos tratamientos alcanzó entre 48 y 55\%. El ABCPE en todos los tratamientos difirió estadísticamente del testigo sin sanear, 
aunque el Biopreparado P2 quedó intermedio, ya que difirió del resto de los tratamientos, aunque también del testigo. En cuanto a la antracosis en flores todos los tratamientos tuvieron menos incidencia que el testigo sin sanear destacándose Caldo Rizósfera, M6, Biopreparado de Meconio y Dithane. En cuanto al ABCPE todos los tratamientos alcanzaron menores valores que el testigo (Tabla 4).

TABla 4. RESUltado DEL ANÁlisis ESTADÍSTiCO PARA LA ENFERMEDAD ANTRACNOSIS CAUSADA POR EL HONGO COLlETOTRICHUM GLOEOSPORIOIDES EN FRUTOS Y FLORES SUGÚN LOS DIFERENTES TRATAMIENTOS.

\begin{tabular}{|l|l|l|l|l|}
\hline & \multicolumn{2}{|c|}{ frutos } & \multicolumn{2}{c|}{ flores } \\
\hline Tratamientos & $\begin{array}{c}\text { Incidencia } \\
(\%)\end{array}$ & \multicolumn{1}{|c|}{ Abcpe } & $\begin{array}{c}\text { Incidencia } \\
(\%)\end{array}$ & ABCPE \\
\hline T. saneado & $55 \mathrm{bc}$ & $5124,00 \mathrm{~b}$ & $47 \mathrm{bc}$ & $4968,25 \mathrm{c}$ \\
\hline $\begin{array}{l}\text { Biopreparado } \\
\text { P1 }\end{array}$ & $56 \mathrm{~b}$ & $5656,00 \mathrm{~b}$ & $47 \mathrm{bc}$ & $4989,25 \mathrm{c}$ \\
\hline $\begin{array}{l}\text { Biopreparado } \\
\text { P2 }\end{array}$ & $60 \mathrm{~b}$ & $6123,25 \mathrm{~b}$ & $49 \mathrm{~b}$ & $5297,25 \mathrm{~b}$ \\
\hline $\begin{array}{l}\text { M. de Montaña } \\
\text { (MM) }\end{array}$ & $58 \mathrm{~b}$ & $5944,75 \mathrm{~b}$ & $50 \mathrm{~b}$ & $5192,25 \mathrm{c}$ \\
\hline $\begin{array}{l}\text { C. Rizósfera } \\
\text { (CR) }\end{array}$ & $57 \mathrm{~b}$ & $5838,00 \mathrm{~b}$ & $49 \mathrm{bc}$ & $5054,00 \mathrm{c}$ \\
\hline $\begin{array}{l}\text { Biopreparado } \\
\text { meconio }\end{array}$ & $56 \mathrm{~b}$ & $5304,25 \mathrm{~b}$ & $49 \mathrm{bc}$ & $5019,00 \mathrm{c}$ \\
\hline $\begin{array}{l}\text { M. Eficientes } \\
\text { (M6) }\end{array}$ & $54 \mathrm{bc}$ & $5367,25 \mathrm{~b}$ & $48 \mathrm{bc}$ & $5145,00 \mathrm{c}$ \\
\hline $\begin{array}{l}\text { Fungicida } \\
\text { Dithane }\end{array}$ & $48 \mathrm{c}$ & $5288,50 \mathrm{~b}$ & $40 \mathrm{c}$ & $4525,50 \mathrm{c}$ \\
\hline $\begin{array}{l}\text { Testigo sin } \\
\text { sanear }\end{array}$ & $75 \mathrm{a}$ & $8527,75 \mathrm{a}$ & $66 \mathrm{a}$ & $7304,50 \mathrm{a}$ \\
\hline $\begin{array}{l}\text { Coefici. Variac. } \\
\text { (\%) }\end{array}$ & 20,0 & 20,54 & 19,5 & 21,0 \\
\hline Error Típico* & 0,035 & 212 & 0,04 & 222 \\
\hline
\end{tabular}

* Letras desiguales en las columnas indican diferencia estadísticamente significativa por la prueba de Tukey.

Los tratamientos alternativos difirieron del testigo en cuanto a la incidencia enfermedad en flores con valores entre 40 y $60 \%$, mientras que se registraron entre un 40 y $48 \%$ de frutos afectados por antracnosis, que son valores relativamente altos, ya que conllevaría a pérdidas de $40 \%$ o más de la producción. Por esta razón en momentos de condiciones muy favorables para la enfermedad o alto potencial de inóculo de Colletotrichum, como en las que se desarrolló el experimento sería recomendable realizar tratamientos con fungicidas efectivos en la floración y combinarlos con los alternativos en las etapas de cosecha y disminuir los riesgos de residuos del fruto durante la comercialización.

No hubo diferencia entre los tratamientos para la variable incidencia de la pudrición por el hongo Botritys en los frutos de fresa aunque la enfermedad no alcanzó el 7\% de incidencia. El ABCPE resultó menor para todos los biopreparados con respecto al testigo sin sanear (Tabla 5).
TABla 5. Resultado DEL ANÁLISIS ESTAdÍSTICO PARA LA ENFERMEDAD PUDRICIÓN POR EL HONGO BOTRYTIS CINEREA EN FRUTOS DE FRESA SUGÚN LOS DIFERENTES TRATAMIENTOS.

\begin{tabular}{|l|l|l|}
\hline \multicolumn{1}{|c|}{ Tratamientos } & \multicolumn{1}{c|}{ Incidencia (\%) } & \multicolumn{1}{c|}{ ABCPE } \\
\hline T. saneado & $2,5 \mathrm{a}$ & $186,25 \mathrm{~b}$ \\
\hline Biopreparado P1 & $2,5 \mathrm{a}$ & $218,75 \mathrm{~b}$ \\
\hline Biopreparado P2 & $2,5 \mathrm{a}$ & $218,75 \mathrm{~b}$ \\
\hline M. de Montaña (MM) & $1,3 \mathrm{a}$ & $148,75 \mathrm{~b}$ \\
\hline C. Rizósfera (CR) & $2,5 \mathrm{a}$ & $183,75 \mathrm{~b}$ \\
\hline Biopreparado meconio & $3,8 \mathrm{a}$ & $201,25 \mathrm{~b}$ \\
\hline M. Eficientes (M6) & $1,3 \mathrm{a}$ & $183,75 \mathrm{~b}$ \\
\hline Fungicida Dithane & $2,5 \mathrm{a}$ & $105,00 \mathrm{~b}$ \\
\hline Testigo sin sanear & $6,3 \mathrm{a}$ & $525,00 \mathrm{a}$ \\
\hline Coefici. Variac. (\%) & 6,61 & 16,03 \\
\hline E. Típico* & 0,035 & 38 \\
\hline
\end{tabular}

* Letras desiguales en las columnas indican diferencia estadísticamente significativa por la prueba de Tukey.

Al final del experimento de tratamientos foliares, no hubo diferencia estadísticamente significativa de la incidencia de la mancha bacteriana por Xanthomonas fragariae en las hojas, sin embargo, con relación a la severidad los tratamientos con los biopreparados MM, CR y Dithane mostraron menores niveles de severidad que el testigo sin sanear, aunque otros tratamientos no presentaron diferencia estadísticamente significativa de estos, pero tampoco del testigo absoluto (Tabla $6)$.

TABla 6. RESUltado DEL ANÁLISIS ESTADÍSTICO PARA LA ENFERMEDAD MANCHA BACTERIANA XANTHOMONAS FRAGARIAE EN HOJAS DE FRESA, SEGÚN LOS DIFERENTES TRATAMIENTOS.

\begin{tabular}{|l|l|l|l|l|}
\hline Tratamientos & $\begin{array}{c}\text { Incidencia } \\
(\%)\end{array}$ & $\begin{array}{c}\text { Severidad } \\
(\%)\end{array}$ & $\begin{array}{c}\text { ABCPE } \\
\text { Inc }\end{array}$ & $\begin{array}{c}\text { ABCPE } \\
\text { SEV }\end{array}$ \\
\hline T. saneado & $52 \mathrm{a}$ & $25 \mathrm{cde}$ & $66,15 \mathrm{bc}$ & $22,48 \mathrm{~d}$ \\
\hline $\begin{array}{l}\text { Biopreparado } \\
\text { P1 }\end{array}$ & $48 \mathrm{a}$ & $27 \mathrm{ab}$ & $61,60 \mathrm{cde}$ & $23,38 \mathrm{c}$ \\
\hline $\begin{array}{l}\text { Biopreparado } \\
\text { P2 }\end{array}$ & $52 \mathrm{a}$ & $27 \mathrm{ab}$ & $63,08 \mathrm{bcd}$ & $24,23 \mathrm{bc}$ \\
\hline $\begin{array}{l}\text { M. de } \\
\text { Montaña } \\
\text { (MM) }\end{array}$ & $50 \mathrm{a}$ & $22 \mathrm{e}$ & $57,66 \mathrm{e}$ & $19,21 \mathrm{~d}$ \\
\hline $\begin{array}{l}\text { C. Rizósfera } \\
\text { (CR) }\end{array}$ & $47 \mathrm{a}$ & $23 \mathrm{de}$ & $60,81 \mathrm{de}$ & $20,80 \mathrm{~d}$ \\
\hline $\begin{array}{l}\text { Biopreparado } \\
\text { meconio }\end{array}$ & $51 \mathrm{a}$ & $27 \mathrm{abc}$ & $65,18 \mathrm{bcd}$ & $24,09 \mathrm{bcd}$ \\
\hline $\begin{array}{l}\text { M. Eficientes } \\
\text { (M6) }\end{array}$ & $47 \mathrm{a}$ & $27 \mathrm{abc}$ & $67,28 \mathrm{~b}$ & $24,09 \mathrm{~b}$ \\
\hline $\begin{array}{l}\text { Fungicida } \\
\text { Dithane }\end{array}$ & $51 \mathrm{a}$ & $25 \mathrm{bcd}$ & $63,96 \mathrm{bcd}$ & $23,15 \mathrm{~cd}$ \\
\hline $\begin{array}{l}\text { Testigo sin } \\
\text { sanear }\end{array}$ & $53 \mathrm{a}$ & $28 \mathrm{a}$ & $72,62 \mathrm{a}$ & $29,75 \mathrm{a}$ \\
\hline $\begin{array}{l}\text { Coefici. } \\
\text { Variac. (\%) }\end{array}$ & 2,5 & 9 & 7,4 & 20 \\
\hline Error Típico* & 0,031 & 0,015 & 1,05 & 0,34 \\
\hline
\end{tabular}

* Letras desiguales en las columnas indican diferencia estadísticamente significativa por la prueba de Tukey. 
El análisis de la ANOVA mostró que para la enfermedad Fusarium sp. se pudo observar que se logró menor porcentaje de incidencia con la aplicación de los biopreparados MM, CR y M6 y los productos Benomil $\mathrm{y}$ fostito de potasio al diferir del testigo (25,62\%), lo cual no ocurrió con el tratamiento con Trichoderma. Similar situación desde el punto de vista estadístico se presentó para el ABCPE de Fusarium sp. Para Phytophthora fragariae, se verificó que el valor de incidencia fue mayor para el testigo $(36,25 \%)$, con diferencia estadísticamente significativa con CR y M6 quedando MM, Benomil, Trichoderma y fosfito de potasio intermedios desde el punto de vista estadístico, pero con diferencia estadísticamente significativa también con el testigo. El ABCPE fue menor para la dinámica de la enfermedad por $P$. fragariae para los tratamientos CR, M6, MM y Benomil (Tabla 7).

TABla 7. Resultado DEL ANÁlisis Estadístico PARA LAS ENFERMEDADES RADICULARES FusARIUM SP. Y Phytophthora FRAGARIAE SUGÚN LOS DIFERENTES TRATAMIENTOS.

\begin{tabular}{|l|l|l|l|l|}
\hline & \multicolumn{2}{|c|}{ Fusarium sp } & \multicolumn{2}{c|}{$\begin{array}{c}\text { Phytophthora } \\
\text { fragariae }\end{array}$} \\
\hline Tratamientos & $\begin{array}{c}\text { Incidencia } \\
\text { (\%) }\end{array}$ & ABCPE & $\begin{array}{c}\text { Incidencia } \\
\text { (\%) }\end{array}$ & ABCPE \\
\hline $\begin{array}{l}\text { Caldo } \\
\text { Rizósfera (CR) }\end{array}$ & $5,00 \mathrm{bc}$ & $376,25 \mathrm{bc}$ & $7,50 \mathrm{~d}$ & $498,75 \mathrm{c}$ \\
\hline $\begin{array}{l}\text { Micror. de } \\
\text { Montaña } \\
\text { (MM) }\end{array}$ & $2,50 \mathrm{c}$ & $157,50 \mathrm{c}$ & $11,25 \mathrm{bcd}$ & $700,00 \mathrm{bc}$ \\
\hline $\begin{array}{l}\text { M. Eficientes } \\
\text { (M6) }\end{array}$ & $5,00 \mathrm{bc}$ & $402,50 \mathrm{bc}$ & $9,375 \mathrm{~cd}$ & $581,87 \mathrm{bc}$ \\
\hline Trichoderma & $13,12 \mathrm{ab}$ & $861,87 \mathrm{ab}$ & $18,75 \mathrm{~b}$ & $936,25 \mathrm{~b}$ \\
\hline $\begin{array}{l}\text { Fungicida } \\
\text { Benomil }\end{array}$ & $5,00 \mathrm{bc}$ & $315,00 \mathrm{bc}$ & $13,12 \mathrm{bcd}$ & $669,37 \mathrm{bc}$ \\
\hline $\begin{array}{l}\text { Fosfito de } \\
\text { potasio } \\
\text { (fungicida) }\end{array}$ & $6,25 \mathrm{bc}$ & $525,00 \mathrm{bc}$ & $15,0 \mathrm{bcd}$ & $936,25 \mathrm{~b}$ \\
\hline Testigo & $25,62 \mathrm{a}$ & $1430,62 \mathrm{a}$ & $36,25 \mathrm{a}$ & $1627,50 \mathrm{a}$ \\
\hline $\begin{array}{l}\text { Coefici. Variac. } \\
\text { (\%) }\end{array}$ & 15 & 20,1 & 12 & 19.9 \\
\hline Error Típico* & 0,07 & 125,54 & 0,05 & 93,7 \\
\hline
\end{tabular}

* Letras desiguales en las columnas indican diferencia estadísticamente significativa por la prueba de Tukey.

Los bioproductos Caldo Rizósfera y MM mostraron resultados similares que al fungicida protectante Dithane contra mancha por Ramularia, la antracnosis en hojas, flores y frutos, la pudrición por Botrytis y la mancha bacteriana.

Para estas enfermedades foliares las posibilidades de los biopreparados de mayor efecto MM y CR sería su incorporación en los momentos de periodos prolongados de cosecha para evitar la contaminación alta de los frutos que van al mercado con residuos químicos y hacer un manejo con los fungicidas en rotación con los productos sistémicos de mayor acción (derivados de los triazoles o de las estrobirulinas), en los periodos en que la fresa pasa a un periodo de recuperación vegetativa para reducir la fuente de inóculo.

Los biopreparados Caldo Rizósfera, ME y M6 resultaron similares al fungicida sistémico Benomil y a fosfito de potasio para el control de las enfermedades radiculares y mantuvieron los porcentajes de incidencia de Fusarium sp. en niveles iguales o inferiores al 5\% (CR y M6) y de Phytophthora fragarie por debajo de $10 \%$ de incidencia (CR, MM y M6).

Los porcentajes de plantas de fresa con síntomas de carencia de fósforo $(\mathrm{P})$ se redujeron con relación al testigo y también en relación a la aplicación foliar del fertilizante que contenía $\mathrm{P}_{2} \mathrm{O}_{5}$ en los tratamientos donde se aplicó M6 y CR. De forma similar ocurrió con el porcentaje de severidad de los síntomas de carencia de $\mathrm{P}$ cuando se aplicó M6 y CR, solo que para esta variable el tratamiento con la aplicación de $\mathrm{P}_{2} \mathrm{O}_{5}$, si fue mejor que el testigo. El contenido promedio de $\mathrm{P}$ foliar fue estadísticamente mayor en los tratamientos con CR y M6 con relación al resto (Tabla 8).

TABLA 8. RESULTADO DEL ANÁLISIS ESTADÍSTICO PARA LOS SÍNTOMAS DE CARENCIA FOLIAR DE FÓSFORO Y LA CONCENTRACIÓN DE FÓSFORO EN HOJAS (P205) SEGÚN LOS DIFERENTES TRATAMIENTOS.

\begin{tabular}{|l|l|l|l|}
\hline & \multicolumn{2}{|c|}{$\begin{array}{c}\text { síntomas de carencia foliar } \\
\text { de fósforo }\end{array}$} & $\begin{array}{c}\text { Concentración } \\
\text { foliar de P }\end{array}$ \\
\hline & \multicolumn{1}{|c|}{ Incidencia } & \multicolumn{1}{|c|}{ Severidad } & \multicolumn{1}{c|}{ P205 } \\
\hline Tratamientos & $\%$ & $\%$ & $\mathrm{mg} / \mathrm{Kg}$ \\
\hline Testigo & $100,0 \mathrm{a}$ & $63,5 \mathrm{a}$ & $9,5 \mathrm{c}$ \\
\hline Biopreparado P1 & $95,0 \mathrm{~b}$ & $37,0 \mathrm{de}$ & $17,7 \mathrm{c}$ \\
\hline Biopreparado P2 & $92,5 \mathrm{~b}$ & $42,0 \mathrm{~cd}$ & $21,2 \mathrm{bc}$ \\
\hline $\begin{array}{l}\text { M. de Montaña } \\
\text { (MM) }\end{array}$ & $92,5 \mathrm{~b}$ & $48,5 \mathrm{bc}$ & $22,09 \mathrm{bc}$ \\
\hline $\begin{array}{l}\text { Caldo Rizósfera } \\
\text { (CR) }\end{array}$ & $32,5 \mathrm{c}$ & $7,0 \mathrm{f}$ & $35,3 \mathrm{a}$ \\
\hline B. meconio & $95,0 \mathrm{~b}$ & $55,5 \mathrm{ab}$ & $21,3 \mathrm{bc}$ \\
\hline $\begin{array}{l}\text { M. Eficientes } \\
\text { (M6) }\end{array}$ & $30,0 \mathrm{c}$ & $6,0 \mathrm{f}$ & $33,3 \mathrm{ab}$ \\
\hline $\begin{array}{l}\text { P2O5 Foliar } \\
\text { (Folyfos) }\end{array}$ & $85,0 \mathrm{a}$ & $26,5 \mathrm{e}$ & $16,7 \mathrm{c}$ \\
\hline C. Variación (\%) & 10,8 & 9,9 & 20,01 \\
\hline Error Típico* & 0,08 & 0,05 & 2,68 \\
\hline
\end{tabular}

" Letras desiguales en las columnas indican diferencia estadísticamente significativa por la prueba de Tukey.

Caldo Rizósfera y M6 disminuyeron en mayor medida la incidencia y severidad de la deficiencia de fósforo y favorecieron una mayor concentración de fósforo en las hojas por lo que estos bioproductos que ya resultaron efectivos para hongos del suelo causante de afectaciones radiculares y en determinada mediada contra enfermedades foliares, al incluirse en un programa de manejo en el cultivo de la fresa pueden jugar varias funciones desde el punto de vista fitosanitario y nutricional. 
Una caracterización microbiología de los principales grupos taxonómicos de los biopreparados que produce ASPAGRO, puso de manifiesto que en MM predominan los actinomicetos, en CR las bacterias y en M6 hongos y bacterias, y que tienen como factor común que todos contienen actinomicetos y bacterias solubilizadoras de $\mathrm{P}$ [4].

Se plantea que el Caldo Rizósfera aporta una gran cantidad y diversidad de microorganismos benéficos que son importantes para la nutrición balanceada de la planta y su defensa contra los fitopatógenos [22], mientras que en otro resultado se informó que en un análisis microbiológico que Caldo Rizósfera tenía mayor población de hongos y de bacterias totales, fijadoras de $\mathrm{N}_{2}$ y solubilizadoras de fósforo que otros preparados [12].

Los actinomicetos controlan hongos y bacterias patogénicas y también aumentan la resistencia de las plantas, mediante un mecanismo de producción de antibióticos que provocan inhibición de patógenos del suelo y benefician el crecimiento y la actividad de Azotobacter y de las micorrizas [23].

La diversidad microbiana y los procesos en el suelo son llevados a cabo por "consorcios microbianos" cuya característica principal es la diversidad funcional más que la de grupos taxonómicos [24], mientras que por otra parte al caracterizar un biopreparado líquido fermentado anaeróbicamente a partir de excreta fresca, se observó que entre sus atributos no estaba la cantidad de microorganismos, sino el contenido de N, P, K y micronutrientes, aunque utilizaron en el proceso un inoculante de un consorcio microbiano de bacterias ácido láctico, que contiene bacterias probióticas del género Lactobacillus, Streptococcus y Bifidobacterium [25].

Algunos actinomicetos han sido descritos como agentes de biocontrol por la capacidad de producir enzimas biodegradativas como quitinasas, glucanasas, peroxidasas y otras [26].

Entre los patógenos de la fresa donde se han destacado la acción de los antagonistas está Botrytis cinerea pudiéndose mencionar la eficacia de Trichoderma, Bacillus subtilis y un complejo biológico a base de bacterias benéficas similar a los resultados obtenidos en el presente estudio [18], así como en otra investigación donde se evaluaron a Trichoderma harzianum y a $\mathrm{T}$. lignorum contra $\mathrm{B}$. cinérea en fresa y se redujo la incidencia y la severidad de la enfermedad [27].

Los géneros bacterianos con mayores potencialidades de uso como solubilizadores de $\mathrm{P}$ son Pseudomonas y Bacillus debido ya que sus principales mecanismos de acción incluyen la producción de ácidos orgánicos, la quelación de los elementos responsables de la insolubilidad de los fosfatos presentes y asimilación directa de fosfatos insolubles [28], por lo que los presentes resul- tados apoyan éstos a partir de los microorganismos encontrados cuando se caracterizó a M6 y CR [4].

Con relación al control de babosas con tierras de diatomeas, a los 7 días de la primera aplicación la TD a $4 \mathrm{~kg} /$ ha causó un $43,48 \%$ de mortalidad y la cal agrícola un $45,65 \%$ sin diferencia estadísticamente significativa con el tratamiento de la TD a $4 \mathrm{~kg} / \mathrm{ha}$, sin embargo, estos tres tratamientos superaron al de TD a $2 \mathrm{~kg} / \mathrm{ha}$. A los siete días después de la segunda aplicación los menores porcentajes de mortalidad se observaron en la TD a los $8 \mathrm{~kg} / \mathrm{ha}$ con $92,38 \%$ (Tabla 9), el resto de tratamientos quedaron por debajo de este estadísticamente, aunque en todas las mortalidades superaron el 60\% establecido para medios biológicos o alternativos por el Centro Nacional de Sanidad Vegetal de Cuba [29].

TABla 9. EFICACIA DE LA TIERRA DE DIATOMEA (TD) CONTRA BABOSAS EN CONDICIONES DE CAMPO A LOS SIETE DÍAS DESPUÉS DE CADA APLICACIÓN. =

\begin{tabular}{|l|l|l|}
\hline & \multicolumn{1}{|c|}{ Mortalidad (\%) } & \multicolumn{1}{|c|}{ Mortalidad (\%) } \\
\hline Tratamientos & Primera aplicación & Segunda aplicación \\
\hline TD $2 \mathrm{~kg} / \mathrm{ha}$ & $35,22 \mathrm{a}$ & $79,52 \mathrm{~b}$ \\
\hline TD $4 \mathrm{~kg} / \mathrm{ha}$ & $44,35 \mathrm{a}$ & $86,52 \mathrm{~b}$ \\
\hline TD $8 \mathrm{~kg} / \mathrm{ha}$ & $43,48 \mathrm{a}$ & $95,24 \mathrm{a}$ \\
\hline Cal Agrícola 4 kg/ha & $45,65 \mathrm{a}$ & $82,38 \mathrm{~b}$ \\
\hline C.V. (\%) & 3,051 & 1,076 \\
\hline Error Típico* & 0,103 & 0,080 \\
\hline
\end{tabular}

* Letras desiguales en las columnas indican diferencia estadísticamente significativa por la prueba de Tukey.

Los resultados mostraron superioridad de la dosis más alta de TD $(8 \mathrm{~kg} / \mathrm{ha})$ con respecto a su persistencia en el tiempo ya que desde los tres días manifestaba más de $90 \%$ de mortalidad, por lo que se refleja que hay un efecto acumulativo de la tierra de diatomea en la segunda aplicación con respecto a la primera. A esto hay que adicionar el efecto positivo que pudo haber jugado el PEGAL como adherente, porque durante la semana de evaluación o posterior se presentaron lluvias en el área del experimento.

Algunos investigadores al realizar las evaluaciones contra moluscos en condiciones de campo con seis extractos de las especies botánicas sobrepasan el $70 \%$ de mortalidad a las 72 horas [30], mientras que otros resultados también hacen referencia de la necesidad de realizar una segunda aplicación con jugos de plantas de la familia agavácea ricos en saponinas para lograr mayor eficacia de los tratamientos contra los moluscos en condiciones de organopónicos [31].

La aplicación en el cultivo de la fresa de la TD puede tener efectos adicionales por su alto contenido de $\mathrm{SiO}_{2}$, [32] que puede conferir un efecto de resistencia de la planta al ataque de insectos como han planteado algunos investigadores [33], [34]. 


\section{Conclusiones}

Los presentes resultados demuestran que es posible sustituir las aplicaciones de fungicidas químicos para el control de enfermedades causadas por hongos del suelo en la fresa y parcialmente los fungicidas foliares con biopreparados como MM, M6 y CR, de igual forma puede atenuarse la carencia de fósforo con algunos de los biopreparados locales (M6 y CR), así como controlar las babosas con tierra de diatomeas, lo que permite incorporar nuevas alternativas para que sean validadas en la producción más limpia del cultivo de fresa en las condiciones de Pamplona (Norte de Santander, Colombia).

\section{REFERENCIAS}

[1] CCB, Programa de apoyo agrícola y agroindustrial vicepresidencia de fortalecimiento empresarial. Bogotá, D.C.: CCB, 2015.

[2] UC, Guía para el manejo de las plagas: Fresas. Oakland: UC, 2005. Available: http://www.oregonstrawberries.org/ fmr/fact_sheets/Guia__Fresas_Espanol.pdf

[3] T. Cano, "Estrategias biológicas para el manejo de enfermedades en el cultivo de fresa (Fragaria ssp)", Colombian J. Horticultural Sci, vol. 7, no. 2, pp. 263-276, Jul. 2013. https://doi.org/10.17584/rcch.2013v7i2.2240

[4] L. Castellanos, N. Céspedes, A. Sequeda, J. E. Jaime \& L. J. Niño, "Caracterización microbiológica de seis biopreparados artesanales", Revi Cient Agroecosist, vol. 6, no. 3, pp. 57-65, Sept. 2018. Available: https://aes.ucf. edu.cu/index.php/aes/article/view/219

[5] G. Mixquititla \& O. G. Villegas, "Importancia de los fosfatos y fosfitos en la nutrición de cultivos", Acta Agrícola y Pecuaria, vol. 2, no. 3, pp. 55-61, Dec. 2016. Available: http://riaa.uaem.mx/handle/20.500.12055/57

[6] O. Saucedo, R. Mello, L. Castellanos, E. Nahas, C. N. Silva, G. Pereira \& L. C. Assis, "Efecto de la fertilización fosfatada con cachaza sobre la actividad microbiana del suelo y la absorción de fósforo en caña de azúcar (Saccharum spp.)", Rev. FCA Uncuyo, vol. 47, no. 1, pp. 33-42. Jun. 2015. Available: https://bdigital.uncu.edu.ar/7468

[7] L. F. Díaz, P. A. Dávalos, A. E. Jofre \& T. O. Martínez, Fresa, deficiencias y sintomas nutricionales. Una guía visual para fertilizar. Celaya: INIFAP, 2017.

[8] R. Escobar. "Las prácticas agrícolas y su incidencia en la calidad y productividad de fresas. (Fragaria vesca, variedad Albión)", in III Congr. Científico Intl, Santo Domingo, 2015. Available: http://www.uniandes.edu.ec/ web/wp-content/uploads/2016/04/Las-pr\%C3\%A1cticasagr\%C 3\%ADcolas-y-su-incidencia-en-la-calidad-yproductividad-de-fresas-Fragaria-vesca-variedadAlbi\%C3\%B3n.pdf

[9] R. Ortiz, J. Villadiego \& C. Cardona, "Valoración de los impactos ambientales totales generados por el uso de plaguicidas en actividades ganaderas en el municipio de Pamplona Norte de Santander, Colombia". Rev Did Amb vol. 7, no. 10, pp. 62-80, Dec. 2011. Available: http://www. didacticaambiental.com/revista/numero10/7\%20valoracion\%20de\%20los\%20impactos.pdf

[10] BID, Manual práctico de uso de EM. Convenio Fondo Especial de Japón. BID ATN/JO-10792 UR. Montevideo, UR: OISCA, Jul. 2009. Available: https://www.emuruguay.org/images/Manual_Practico_Uso_EM_OISCA_ BID.pdf
[11] L. Castro-Barquero, M. Murillo-Roos, L. Uribe-Lorío \& R. Mata-Chinchilla, "Inoculación al suelo con Pseudomonas fluorescens, Azospirillum oryzae, Bacillus subtilis y Microorganismos de Montaña (MM) y su efecto sobre un sistema de rotación soya-tomate bajo condiciones de invernadero", Agron. Costarricense, vol. 39, no. 3, pp. 21-36, Jan. 2015. Available: http://hdl.handle. net/10669/74551

[12] S. E. Viteri, M. Granados \& A. R. González, "Potencial de los caldos rizósfera y súper cuatro como biofertilizantes para la sostenibilidad del cultivo de cebolla de bulbo (Allium cepa)", Agron. Col, vol. 26, no. 3, pp. 517-524, Sept. 2008.

[13] L.Marco\&R. E. Reyes, “Tecnologías limpias aplicadas a la agricultura", Interciencias, vol. 28, no. 5, may, 2003. [Online]. Available: http://ve.scielo.org/scielo.php?script=sci arttext\&pid=S0378-18442003000500002

[14] M. Romero \& E. M. Suárez, Huella ambiental del cultivo de la fresa. Granada: IFAPA, 2018. Available: https:// www.interempresas.net/Horticola/Articulos/230272Huella-ambiental-del-cultivo-de-la-fresa.html

[15] M. A. Amézquita. "Niveles de "bocashi" y "microorganismos eficaces" en el rendimiento de fresa (Fragaria $\mathrm{x}$ ananassa Duch) cv. Selva en condiciones de zonas áridas - irrigación majes", Degree Thesis, Fac. Agronomía. UNSA, Arequipa, Perú, 2018. Available: http://Repositorio.Unsa.Edu.Pe/Handle/Unsa/6141

[16] J. S. Bolívar, E. H, Pinzón \& G. E Cely, "Efecto de sustratos orgánicos en plantas de fresa (Fragaria sp.) cv 'Albión' bajo condiciones de campo", Rev. Cienc Agricul, vol. 13, no. 2, pp. 19-28, Nov. 2016. https://doi. org/10.19053/01228420.v13.n2.2016.5548

[17] F. A. Chiqui \& M. Lema. "Evaluación del rendimiento en el cultivo de fresa (Fragaria sp.) variedad oso grande, bajo invernadero mediante dos tipos de fertilización (orgánica y química) en la parroquia Octavio Cordero Palacios, Cantón Cuenca”, Degree Thesis, Fac. Ciencias Agropecuarias y Ambientales, Univ. Politécnica Salesiana, Cuenca, 2010. Available: https://dspace.ups.edu.ec/ bitstream/123456789/4745/1/UPS-CT001855.pdf

[18] A. P. Quezada. "Evaluación del comportamiento de fungicidas microbiológicos en la prevención de Botrytis en el cultivo de fresa (Fragaria vesca)", M.S. Thesis, Fac. Ing. Agronómica, Univ. Técnica de Ambato, Ecuador, 2011. Available: https://repositorio.uta.edu.ec/handle/123456789/1680

[19] E. C. Niño \& O. Guerrero, "Efecto de Trichoderma lignorum y Sacharomyces cerevisiae en el control del hongo Botrytis Cinerea causante del moho gris de la fresa y su rendimiento, en el municipio de Subachoque Cundinamarca", Inventum, vol. 11, pp. 1-20, Jul. 2011. http://dx.doi. org/10.26620/uniminuto.inventum.6.11.2011.12-20

[20] Ciba-Geigy S. A., Manual para ensayos de campo en producción vegetal, 2 Ed, Basilea: Ciba-Geigy S.A., 1981.

[21] C. L. Campbell \& L. V. Madden, Introduction to Plant Disease Epidemiology. New York, USA: Wiley, 1990

[22] M. J. Méndez \& S. E. Viteri, "Alternativas de biofertilización para la producción sostenible de cebolla de bulbo (Allium cepa) en Cucaita, Boyacá", Agron. Colombiana, vol. 25, no. 1, pp. 168-175, Jan. 2007. Available: https://repository.agrosavia.co/bitstream/handle/20.500.12324/18279/43049_49258. pdf? sequence $=1 \&$ isAllowed $=y$

[23] F. M. Coutinho, "Divulgação das Plantas Medicinais, da Homeopatia e da Produção de Alimentos Orgânicos" in Cuaderno los Microrganismos Eficientes Instruções práticas sobre uso Ecológico e social do EM. Viçosa: UFV, 2008. 
[24] M. G. Kibblewhite, K. Ritz \& M. J. Swift, "Soil health in agricultural systems", Philos. T. R. Soc. B, vol. 363, no. 1492, pp. 685-701, Sept. 2015. https://doi.org/10.1098/ rstb.2007.2178

[25] L. Peralta, J. Juscamaita \& V. Meza, “Obtención y caracterización de abono orgánico líquido a través del tratamiento de excretas del ganado vacuno de un establo lechero usando un consorcio microbiano ácido láctico", Ecología Aplicada, vol. 15, no. 1, pp. 1-10, Jan. 2016. https://doi.org/10.21704/rea.v15i1.577

[26] M. V. Arasu, G. A. Esmail, N. A. Al-Dhabi \& P. Karuppiah, "Managing pests and diseases of grain legumes with secondary metabolites from actinomycetes" in Plant growth promoting actinobacteria: A new avenue for enhancing the productivity and soil fertility of grain legumes. Singapore: Springer, 2016. https://doi. org/10.1007/978-981-10-0707-1_6

[27] J. B. Merchán-Gaitán, R. L. Ferrucho \& J. G. Álvarez, "Efecto de dos cepas de Trichoderma en el control de Botrytis cinerea y la calidad del fruto en fresa. (Fragaria sp.)", Rev Col Cienc Hort, vol. 8, no. 1, pp. 44-56, Jan. 2014. https://doi.org/10.17584/rcch.2014v8i1.2799

[28] G. M. Restrepo, S. Marulanda, Y de la Fe-Pérez, A. Díaz, L. Vera \& A. Hernández, "Bacterias solubilizadoras de fosfato y sus potencialidades de uso en la promoción del crecimiento de cultivos de importancia económica", Rev CENIC Cienc Biol, vol. 46, no. 1, pp. 63-76, Jan. 2015. Available: https://ainfo.cnptia.embrapa.br/digital/bitstream/item/119061/1/Bacterias-solubilizadoras-de-fosfato-y-sus-potencialidades-de-uso-Baldani.pdf

[29] Centro Nacional de Sanidad Vegetal, Manual del Inspector de Protección de Plantas. La Habana: Centro Nacional de Sanidad Vegetal, 2014.

[30] C. Martín, Y. Pérez, L. Castellanos \& B. Soto, "Efectividad de extractos vegetales para el control de Praticolella griseola (Pfeiffer) (Gastropoda: Polygyridae)", Cent $A g r$, vol. 44, no. 2, pp. 68-74, Apr, 2017. Available: http:// cagricola.uclv.edu.cu/descargas/pdf/V44-Numero_2/ cag09217.pdf

[31] M. Nodarse, L. Castellanos, N. Herrera \& M. Morfa, "Acción molusquicida de extractos vegetales de tres especies de la familia Avagaceae contra Praticolella griseola (Pfeiffer)", Rev Prot Veg, vol. 32, no. 2, May. 2017.

[32] M. L. Bilbao, M. O. Mañá \& F. Murúa, "Evaluación del efecto insecticida de tierra de diatomeas sobre Triatoma infestans (Hemíptera, Reduviidae)", Rev Per Med Exp Salud Pública, vol. 24, no. 2, pp. 179-181, Apr. 2007.

[33] L. Castellanos, R. de Mello, G. B. Silva, C. N. Silva, O. Fernández, R. Pereira, L. Rosatto \& R. Alvárez, "Daños por Spodoptera frugiperda Smith en maíz en función de nitrógeno, potasio y silicio", Rev. Protec. Veg., vol. 30, no. 3, Sept. 2015. Disponible en http://200.14.50.70/index. php/RPV/article/view/605

[34] L. Castellanos, C. N. Silva \& R. de Mello, "Silicon in the crop resistance to agricultural pest", Cultivos Tropicales, vol. 36, no. Suppl. 1, pp. 16-24, Jan. 2015. Available: http://ediciones.inca.edu.cu/index.php/ediciones/article/ view/1112
Leónides Castellanos González es Ingeniero agrónomo de la Universidad Central de Las Villas (Cuba). Magister en Ciencias Agrícolas de la Universidad Agraria de la Habana (Cuba) y Dr. En Ciencias Agrícolas en la Universidad Central de Las Villas (Cuba). Posdoctorado en Metodología de la investigación científica en la Universidad de Cienfuegos (Cuba) y en Nutrición de Plantas en la UNESP (Brasil). Investigador Senior de Colciencias. Profesor de la Universidad de Pamplona (Colombia). http://orcid.org/0000-00019285-4879

Nestor Céspedes Novoa es Licenciado en Recursos Naturales de la Granja Agrobiológica Sol Vida, ASPAGRO, en Pamplona (Norte de Santander, Colombia).

Alex Valdovino Sanjuan es Ingeniero Agrónomo y profesor en la Universidad de Pamplona (Norte de Santander, Colombia). 